\section{Informationskampagne „ADHS und Zukunftsträume" gestartet}

\begin{abstract}
— Anfang April fiel in Berlin der Startschuss für die Informationskampagne „ADHS und Zukunftsträume", initiiert von der Shire Deutschland GmbH (www.adhs-zukunftstraeume.de). Und gleich in der Auftaktveranstaltung wurde deutlich: Aufklärung ist dringend notwendig, vor allen mit Blick auf die Diagnostik. „,ADHS ist keine Modekrankheit", betonte Bundesfamilienministerin a. D. Renate Schmidt, die Schirmherrin der Kampagne. Mangelhaftes diagnostisches Vorgehen führt in Deutschland allerdings zu einer paradoxen Situation: Es gibt zu viele falschpositive Befunde, gleichzeitig aber zu viele Kinder mit ADHS, die nicht diagnostiziert werden.

Als einen der Gründe nannte Prof. Martin Holtmann aus Hamm die zu geringe Zahl spezialisierter Kinderärzte und Kinder- und Jugendpsychiater.„Eine frühzeitige und um-
\end{abstract}

fassende Diagnostik durch einen spezialisierten Arzt gemäß den medizinischen Leitlinien ist aber entscheidend für eine erfolgreiche Behandlung", betonte er. Es dürfe nicht sein, dass die Diagnose „ADHS“ aufgrund eines einzigen Fragebogentests gestellt werde. Wichtig sei, dass das Kind nicht nur Symptome habe, sondern dadurch auch beeinträchtigt sei. Und dass diese in allen Lebensbereichen auftreten.

Die Informationskampagne hat das Ziel, durch umfassende Aufklärung das Wissen über ADHS zu verbessern und "einen positiven Blick auf die Betroffenen zu entwickeln", so Schmidt. Sie sollen „ihre Zukunftsträume in echte Zukunftsräume" verwandeln können.

Dr. Beate Fessler

Pressekonferenz ,ADHS - Mode oder Krankheit?" Berlin, 5. April 2011. Veranstalter: Shire

\title{
Hemmkörper-Hämophilie: neue Dosisstärke beschleunigt Behandlung
}

- Den rekombinanten aktivierten Gerinnungsfaktor VII (rFVIla, Eptacog alfa [aktiviert], NovoSeven ${ }^{\oplus}$ ) gibt es seit März 2011 in einer zusätzlichen Dosisstärke: Durchstechflaschen mit 8 mg ergänzen damit das bisherige Angebot von $1 \mathrm{mg}, 2 \mathrm{mg}$ und $5 \mathrm{mg}$. Die neue Dosierung soll Hämophilie-Patienten, die höhere Dosen benötigen, eine schnellere Zubereitung und damit eine frühere Behandlung mit dem Faktor ermöglichen.„Die schnellere Rekonstitution bringt eine Zeitersparnis von bis zu 66\%", berichtete Novo Nordisk-Mitarbeiterin Kristina Saala, Mainz. Die einzelnen Dosen sind für Medikation und Lösungsmittel zur sicheren und schnellen Identifikation durch unterschiedliche Farbkodierungen gekennzeichnet. Mit Genehmigung der amerikanischen und europäischen Zulassungsbehörden wurde die Haltbarkeitsdauer um ein Jahr auf jetzt drei Jahre verlängert. Stabilitätsstudien hatten für die vorliegende, nicht veränderte Formulierung gezeigt, dass auch eine dreijährige Lagerung bei $25^{\circ} \mathrm{C}$, ebenso wie die entsprechende Lagerung im Kühlschrank, die Stabilität des Präparates nicht beeinträchtigt.
Faktor VII wird als Bypass-Therapie zur Behandlung und Prophylaxe von Blutungen eingesetzt bei Patienten mit Hämophilie $A$ und Hämophilie $B$, bei denen Antikörper (Hemmkörper) den substituierten Faktor VIII beziehungsweise IX inaktivieren. Bei einer Blutung appliziert der Patient Faktor VII als intravenösen Bolus - entweder in der Dosis von $90 \mu \mathrm{g} / \mathrm{kg} \mathrm{KG}$ in Intervallen von zwei bis drei Stunden - oder bei leichten und mittelschweren Blutungen als Einzelbolus von $270 \mu \mathrm{g} / \mathrm{kg} \mathrm{KG}$. Eine weitere Indikation ist der autosomal-rezessiv vererbte Faktor-VII-Mangel, der beide Geschlechter gleich häufig betrifft, mit einer Inzidenz von 1:300.000500.000 jedoch selten ist. Hier erfolgt die Intervalltherapie bei Blutung mit 15-30 $\mu \mathrm{g} /$ kg KG alle vier bis sechs Stunden bis die Hämostase erreicht ist.

Michael Koczorek

Presse-Briefing „Schnelle Blutungskontrolle bei Hämophilie mit Hemmkörpern mit dem neuen NovoSeven 8 mg", im Rahmen der 55. Jahrestagung der Gesellschaft für Thrombose- und Hämostaseforschung (GTH). Wiesbaden, 16. Februar 2011. Veranstalter: Novo Nordisk, Mainz
ÖKO-TEST-Note „gut" für

Schleimlöser

Der pflanzliche Schleimlöser GeloMyrtol ${ }^{\circledR}$ forte wurde von der Zeitschrift ÖKO-TEST mit dem Gesamturteil "gut" ausgezeichnet. In der Juni-Ausgabe bewertet ÖKOTEST im Rahmen der Serie "Naturarzneimittel" insgesamt 17 pflanzliche Hustenmittel. GeloMyrtol ${ }^{\circledR}$ forte überzeugte durch seine gute schleimlösende Wirkung und gehört damit zu den Siegern der Bewertung. Der Schleimlöser hat sich sowohl bei akuten als auch bei chronischen Entzündungen der Atemwege bewährt. Das ätherische Öl Myrtol standardisiert wirkt

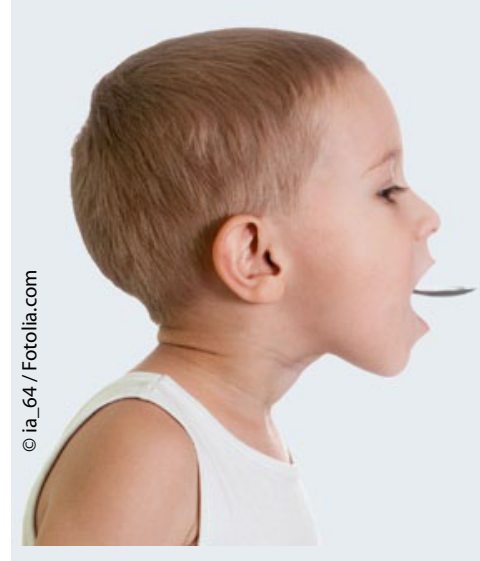

direkt am Infektionsherd, beschleunigt dort das Abschwellen der Schleimhäute und löst das zähe, festsitzende Sekret. Zusätzlich besitzt es antientzündliche, antibakterielle und antioxidative Eigenschaften.

Nach Informationen von Pohl-Boskamp, Hohenlockstedt

\section{Zulassung bei PAH}

Pfizer hat für sein Arzneimittel Revatio ${ }^{\circledR}$ (Sildenafilcitrat) die Zulassung der Europäischen Kommission für die Behandlung pädiatrischer Patienten von einem bis 17 Jahren mit pulmonaler arterieller Hypertonie (PAH) erhalten. Die Wirksamkeit im Sinne einer Verbesserung der körperlichen Belastbarkeit oder pulmonalen Hämodynamik wurde bei der primären pulmonalen Hypertonie sowie bei der pulmonalen Hypertonie aufgrund von angeborenen Herzfehlern nachgewiesen. Die Zulassung stützt sich auf die Ergebnisse einer klinischen Phase-III-Studie, in der Wirksamkeit und Sicherheit von Revatio ${ }^{\circledR}$ im Vergleich zu Placebo bei 234 Kindern und Jugendlichen bestätigt werden konnte.

Nach Informationen von Pfizer, Berlin 